\title{
Blindagens cerâmicas para aplicações balísticas: uma revisão
}

\section{(Ceramic armors for ballistic applications: a review)}

\author{
M. V. da Silval, D. Stainer ${ }^{1}$, H.A.Al-Qureshi ${ }^{2}$, D. Hotza ${ }^{2}$ \\ ${ }^{1,2}$ Construções Mecânicas Cocal (CMC), Av. Valdemar Kleinubing s/ $n^{o}, \mathrm{~km}$ 01, Cocal do Sul, SC 88845-000 \\ Núcleo de Pesquisa em Materiais Cerâmicos e Vítreos - CERMAT \\ ${ }_{2}^{2}$ Programa de Pós-Graduação em Ciência e Engenharia de Materiais - PGMAT, Universidade Federal de Santa \\ Catarina - UFSC Florianópolis, SC 88040-900
}

\begin{abstract}
Resumo
A combinação de propriedades físicas e mecânicas qualificam as cerâmicas para aplicações em sistemas de proteção balística. Materiais cerâmicos normalmente formam a primeira camada de um sistema de blindagem mista, recebendo o impacto inicial do projétil, e dissipando grande parte da energia cinética ao fragmentar o projétil. Os principais materiais cerâmicos para blindagem balística são a alumina, o carbeto de silício e o carbeto de boro. Novas técnicas de processamento de materiais cerâmicos além do modelamento do mecanismo de fratura sob alto impacto energético são áreas que têm sido amplamente investigadas. Desenvolvimentos em particular na área de compósitos de matriz cerâmica, reforçados com fibras ou com base na transformação por tenacificação abrem novas perspectivas de uso e de melhor desempenho de sistemas de proteção balística a base de materiais cerâmicos.
\end{abstract}

Palavras-chave: proteção balística, blindagem, impacto, alumina, carbeto de silício, carbeto de boro, compósitos de matriz cerâmica.

\begin{abstract}
The combination of physical and mechanical properties qualifies ceramic materials for applications in ballistic protection systems. Ceramics usually form the first layer of a mixed shielding system, getting the initial impact of the projectile, and dissipating much of the kinetic energy of the projectile fragments. The main ceramic materials for ballistic armor are alumina, silicon carbide and boron carbide. New processing techniques of ceramic materials as well as modeling the mechanism of fracture under high energy impact are areas that have been widely investigated. Developments particularly in the area of ceramic matrix composites reinforced with fibers or based on transformation toughening open new perspectives for better performance and use of ceramic-based ballistic protection systems.
\end{abstract}

Keywords: ballistic protection, armor, impact, alumina, silicon carbine, boron carbide, ceramic matrix composites.

\section{INTRODUÇÃO}

Materiais cerâmicos têm sido considerados para aplicações de blindagem devido a sua baixa densidade, alta resistência à compressão e dureza [1]. Relatos de uso de cerâmica para aplicações de defesa remontam ao início dos anos 1960, em blindagens de aeronaves e pessoais. O sistema desenvolvido consistia em um compósito de carbeto de boro ligado a fibras de vidro ou aramida, coberto com um tecido de proteção. A substituição de materiais metálicos por materiais cerâmicos garantiu muitas melhorias nas aeronaves, como redução de peso, que por sua vez implica um aumento da autonomia, capacidade de transporte, além de maior capacidade de blindagem [2].

Atualmente, nenhum material apresenta por si só as características requeridas para suportar a combinação de tensões mecânicas provenientes de um evento balístico. Um material cerâmico com elevada resistência à compressão, por exemplo, normalmente apresenta uma baixa resistência à tração, e ao ser submetido ao impacto balístico sofre esforços de tração na superfície oposta ao impacto. Uma solução é a produção de blindagens mistas que combinem propriedades específicas de dois materiais. A blindagem mista é formada pela combinação de dois materiais com propriedades diferentes e complementares responsáveis por evitar a penetração do projétil, seja em um colete, veículo, aeronave ou satélite.

Materiais cerâmicos normalmente formam a primeira camada e são aqueles que recebem o impacto inicial do projétil. Sua função é desgastar a ponta do projétil dissipando grande parte da energia cinética, fragmentando sua massa e melhorando a distribuição da pressão do impacto sobre a segunda camada. A segunda camada da blindagem mista, também chamada de base, é formada por materiais dúcteis e têm como função absorver a energia cinética residual do projétil, dos estilhaços oriundos do projétil e da própria cerâmica através de sua deformação plástica [3]. O requisito mais importante para o material da base é que ele não falhe durante os estágios iniciais do processo de penetração do projétil. Assim, a base deve suportar as tensões de compressão que são transferidas para a cerâmica após o impacto, evitando penetração de estilhaços de projétil e/ 
ou cerâmica com elevada energia cinética ou deformandose excessivamente de maneira a colocar em risco a vida de pessoas por detrás da blindagem, ou a integridade de equipamentos.

Os principais materiais cerâmicos para blindagem balística são a alumina, o carbeto de silício e o carbeto de boro. O carbeto de boro é o que apresenta as melhores propriedades mecânicas: maior dureza, módulo de elasticidade e tenacidade à fratura e a menor densidade, entretanto possui o maior custo de fabricação. $O$ carbeto de silício possui propriedades mecânicas um pouco inferiores ao carbeto de boro, porém apresenta a vantagem de ter um custo inferior. Ambos são utilizados em blindagens para calibres pesados. No entanto, a alumina é normalmente utilizada para calibres leves, mesmo sendo suas propriedades inferiores aos carbetos de boro e de silício, devido a ser mais viável economicamente [2]. Atualmente cerâmicas compósitas também estão sendo utilizados em pesquisas e desenvolvimento de novas blindagens, sendo as cerâmicas de alumina-zircônia tetragonal uma das mais utilizadas.

Neste trabalho, o desempenho balístico será discutido a partir da relação entre estrutura (composição), processamento (particularmente sinterização) e propriedades dos materiais cerâmicos mais comumente utilizados com fins de proteção balística.

\section{Blindagem mista}

No processo de resistência ao impacto de projétil de alta energia, são necessárias as seguintes propriedades: dureza e alta resistência à compressão no início do impacto e resistência à flexão e ductilidade nos estágios finais. A blindagem mista consiste na sobreposição de placas de diferentes materiais com objetivo de utilizar suas diferentes propriedades para se garantir um melhor desempenho da blindagem. Os materiais cerâmicos possuem alta resistência à compressão e alta dureza; entretanto, apresentam baixa resistência à tração e são frágeis. São utilizados para resistir os esforços provenientes dos eventos iniciais. Os materiais dúcteis (metálicos ou poliméricos) são utilizados para resistir o dano causado pelo projétil, por seus fragmentos e pelos fragmentos da cerâmica danificada.

A primeira etapa é bem desempenhada pelos materiais cerâmicos, que suportam a elevada carga de compressão causada pelo projétil, promovem a destruição da ponta do projétil e a deformação do material de fundo. A segunda etapa acontece com a grande tensão de compressão exercida

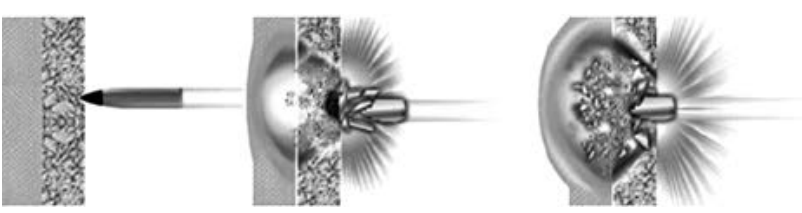

Figura 1: Esquema de blindagem mista: (a) antes; (b) ao longo e (c) depois de impacto balístico.

[Figure 1: Schematic of mixed armor: (a) before; (b) during and (c) after ballistic impact.] pelos materiais cerâmicos ao projétil no instante do impacto que causando a erosão da ponta de projéteis endurecidos ou a deformação da ponta dos projéteis dúcteis. Em ambos os casos, ocorre o aumento de área de contato favorecendo a distribuição da pressão sobre a blindagem. Na terceira etapa, há absorção da energia residual dos fragmentos do projétil e da própria cerâmica através da deformação plástica. Os materiais cerâmicos não são adequados para esta etapa devido a suas restrições previamente discutidas. Para os requisitos de resistência à flexão e ductilidade, os materiais metálicos ou poliméricos possuem melhor desempenho, podendo ser alumínio aeronáutico, fibras de aramida ou aço dúctil de acordo com o fator peso [2]. Os esforços iniciais de compressão sobre a cerâmica causam tensões de tração do lado oposto ao impacto. Sendo a base de material dúctil, esta é capaz de se deformar sem se romper e de suportar as tensões exercidas neste local, evitando a ocorrência de falha. A função principal da base é não falhar durante os estágios iniciais do processo de penetração do projétil, devendo suportar toda tensão de compressão exercida sobre a cerâmica [4].

O projeto de uma blindagem composta deve levar em consideração fatores como peso, nível da proteção desejada, ambiente de operação e danos colaterais [5]. Esses danos podem ser os fragmentos arremessados com alta velocidade durante o impacto do projétil e também a eventual deformação excessiva da base. Entretanto, medidas de segurança solucionam facilmente essa condição. Uma das soluções é aplicar um revestimento externo à cerâmica capaz de conter os fragmentos, como um tecido de aramida. Outra medida é projetar um espaço adequado da blindagem em relação aos itens internos do equipamento a ser protegido. $\mathrm{O}$ desenvolvimento de novos armamentos gera o desafio tecnológico de se produzir novas blindagens, assim como modificar e aperfeiçoar as blindagens mistas já existentes com o intuito de se resistir a essa nova ameaça.

Um número de critérios deve ser considerado quando se selecionam materiais para sistemas de proteções balísticas, incluindo as características da ameaça específica a ser vencida, volume e peso aceitáveis para o sistema e seu custo. Devido à abrangência dos critérios de design dos sistemas de proteção, não se pode considerar um "melhor" material para todas as aplicações. Um exemplo disso são as situações onde o fator peso é restrito tornando determinados materiais inadequados para tal utilização. Por exemplo, blindagens metálicas são muito utilizadas em veículos, mas inadequadas para proteções pessoais.

\section{Cerâmicas balísticas}

Materiais cerâmicos têm sido alvo de pesquisa e desenvolvimento em sistemas de blindagens de peso reduzido e desempenho balístico alto. Mesmo com os recentes progressos nesta área, a compreensão sobre o comportamento balístico de materiais cerâmicos ainda está em pleno desenvolvimento [2].

Uma blindagem cerâmica se constitui de um revestimento 
Tabela I - Propriedades dos materiais e seu papel no desempenho balístico.

[Table I - Material properties and ballistic performance.]

\begin{tabular}{ll}
\hline Propriedade do material & Efeito no desempenho balístico \\
\hline Densidade & Peso do sistema de proteção \\
Dureza & Dano ao projétil \\
Módulo de elasticidade & Propagação das ondas de tensão \\
Resistência mecânica & Resistência a múltiplos impactos \\
Tenacidade à fratura & Resistência a múltiplos impactos, durabilidade em campo \\
Modo da fratura (inter ou transgranular) & Absorção de energia \\
\hline
\end{tabular}

resistente e rígido capaz de resistir aos enormes esforços provenientes de um evento balístico. Para que isso ocorra, é necessário que o material cerâmico apresente valores elevados de resistência mecânica, módulo de elasticidade e dureza [6]. A tenacidade à fratura, que corresponde à energia que o material tem a capacidade de absorver antes que ocorra a sua fratura, é um requisito fundamental para esta aplicação. [7]

Além das principais cerâmicas comerciais já citadas, outros materiais cerâmicos estão em processo de pesquisa e desenvolvimento, atualmente destaca-se o desenvolvimento de compósitos de matriz cerâmica (CMCs), especificamente o sistema $\mathrm{Al}_{2} \mathrm{O}_{3} / \mathrm{ZrO}_{2}$. Os principais motivos pelos quais os materiais cerâmicos não são tão utilizados majoritariamente em blindagens são seu custo elevado, difícil processabilidade e o fato de que é consideravelmente difícil de prever seu desempenho balístico a partir das suas propriedades [7]. Apenas com um bom controle das propriedades relevantes e da microestrutura se pode garantir um bom desempenho balístico.

Atualmente não se pode correlacionar efetivamente desempenho balístico a uma única característica ou propriedade de um material. Isso acontece devido à natureza dinâmica do evento (nano a microssegundos). Assim testes balísticos sob determinadas condições são sempre necessários para se determinar a eficiência dos sistemas de proteção. Entretanto, várias propriedades podem ser associadas a determinados papeis no desempenho balístico, como lista a Tabela I (adaptada [7]).

Todas as propriedades listadas são afetadas diretamente pela microestrutura do material cerâmico, na forma de tamanho de grão, fases cristalinas, transformações de fases e porosidade. Assim sendo o controle microestrutural é extremamente importante no desenvolvimento de uma cerâmica balística, pois as propriedades chaves para o desempenho balístico são dependentes desse.

Cerâmicas não-óxidas como $\mathrm{B}_{4} \mathrm{C}$ e $\mathrm{SiC}$, apresentam um elevado desempenho balístico devido a sua alta dureza e baixa densidade; entretanto, seus custos de matériaprima e processamento são elevados [8]. A alumina é o material de blindagem que apresenta as melhores relações custo-benefício dentre as cerâmicas avançadas: módulo de elasticidade elevado, refratariedade alta, dureza alta, e viabilidade comercial. No entanto, algumas de suas propriedades, como sua baixa tenacidade à fratura e baixa resistência à flexão fazem com que seu desempenho balístico seja inferior ao dos carbetos de silício e boro. As propriedades da alumina podem ser melhoradas, seja por meio da introdução de zircônia em compósitos de matriz cerâmica, que aumentam tenacidade à fratura e a resistência à flexão. Em particular, a introdução de partículas pequenas de zircônia tetragonal policristalina ou de fibras cerâmicas, amplia sua gama de aplicações estruturais $[9,10]$.

$\mathrm{O}$ projeto de uma blindagem utilizando materiais cerâmicos deve considerar que, nos impactos balísticos, a fratura é associada a cargas instantâneas, bem diferentes das com cargas estáticas. Na condição de carga estática, as tensões e deformações são distribuídas ao longo do corpo solicitado e todos os pontos participam do processo de início da fratura. Nas cargas instantâneas, as tensões e deformações encontram-se muito localizadas, de forma que as fraturas podem ocorrer em uma parte isolada do corpo. Esse tipo de impacto pode modificar drasticamente as propriedades mecânicas do material devido às elevadas pressões e as rápidas taxas de carregamento. $\mathrm{O}$ impacto na blindagem composta de cerâmica por projéteis com alta energia cinética normalmente dá origem a um cone de fraturas com trincas radiais e circunferências como mostra a Fig. 2.

Pesquisas foram efetuadas para melhor compreender o mecanismo de impacto e penetração na blindagem mista com cerâmica: Os projéteis disparados contra alvos de cerâmica de alumina cujas pontas eram achatadas apresentaram velocidades residuais maiores após a perfuração dos alvos e, portanto, possuem maior poder de penetração em relação aos projéteis com geometria pontiaguda. Uma única camada de material cerâmico absorve mais energia que várias camadas compondo uma mesma espessura [4]. Imediatamente após o impacto na primeira placa, ocorre a formação de uma trinca axial na interface entre as duas placas, o que provoca a

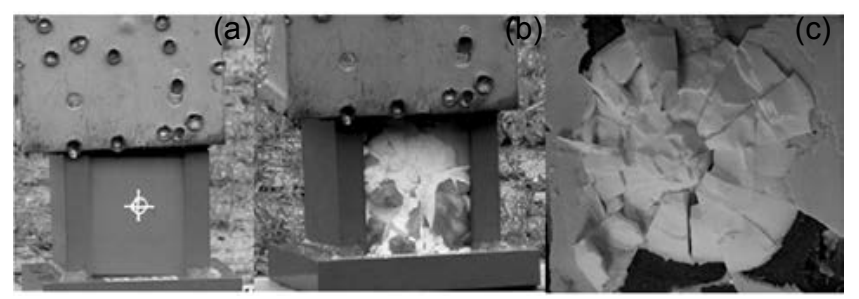

Figura 2: Placa de alumina em ensaio balístico: (a) moldura; (b) depois de impacto e (c) detalhe de fragmentos.

[Figure 2: Alumina plate upon ballistic test: (a) frame; (b) after impact (c) detail of fragments.] 
Tabela II - Cerâmicas balísticas e seu processamento.

[Table II - Ballistic ceramics and their processing.]

\begin{tabular}{|c|c|c|c|}
\hline Processo & Materiais & Vantagens & Desvantagens \\
\hline $\begin{array}{l}\text { Sinterização em estado } \\
\text { sólido ou a vácuo }\end{array}$ & $\begin{array}{l}\text { Hexoloy SiC, Purbide } \mathrm{SiC} \\
\text { MCT SSS SiC, CAP-3 } \mathrm{Al}_{2} \mathrm{O}_{3}\end{array}$ & $\begin{array}{l}\text { Sem contorno de grão, } \\
\text { baixa porosidade }\end{array}$ & $\begin{array}{l}\text { Alta temperatura, } \\
\text { crescimento de grão }\end{array}$ \\
\hline Sinterização em fase líquida & Ekastic-T, MCT LPS SiC & $\begin{array}{l}\text { Baixa temperatura, grãos } \\
\text { finos, baixa porosidade }\end{array}$ & $\begin{array}{l}\text { Contorno de grão } \\
\text { nos óxidos }\end{array}$ \\
\hline $\begin{array}{l}\text { Prensagem a quente } \\
\quad \text { (hot pressing) }\end{array}$ & $\begin{array}{c}\text { Ceralloy B4C, Norbide B4C, } \\
\text { Ceralloy } \mathrm{SiC}, \mathrm{SiC}-\mathrm{N}, \mathrm{TiB}_{2}\end{array}$ & $\begin{array}{l}\text { Baixa temperatura, baixa } \\
\text { porosidade }\end{array}$ & Limitação em formato \\
\hline $\begin{array}{l}\text { Ligação por reação } \\
\text { (reaction bonding) }\end{array}$ & $\begin{array}{c}\mathrm{Si} / \mathrm{SiC}, \mathrm{Si} / \mathrm{B}_{4} \mathrm{C} \\
\text { (MCT RBSC, RBBC) }\end{array}$ & $\begin{array}{l}\text { Baixa temperatura, capaz de } \\
\text { formar estruturas complexas. }\end{array}$ & Silício residual \\
\hline
\end{tabular}

fratura prematura do conjunto. Durante o primeiro estágio do mecanismo de penetração, o fator mais importante é manter a integridade da cerâmica para que possa haver erosão da maior quantidade possível de massa do projétil. Assim, retarda-se o início da fratura do material cerâmico. Esse fator é determinante para a escolha da cerâmica balística a ser utilizada, priorizando cerâmicas de alta dureza e baixa densidade [2].

\section{Processamento de cerâmicas balísticas}

As cerâmicas balísticas tradicionalmente usadas são à base de $\mathrm{Al}_{2} \mathrm{O}_{3}$ sinterizada e $\mathrm{B}_{4} \mathrm{C}$ prensado a quente (hot-pressed), devido a seu custo, facilidade de produção e propriedades satisfatórias. Com o desenvolvimento dos sistemas de proteção para resistir a ameaças de maior energia, a alumina acabou ficando em segundo plano por sua alta densidade, especialmente em proteções pessoais (coletes). Como resultado, a utilização de $\mathrm{B}_{4} \mathrm{C}$ e $\mathrm{SiC}$ se tornou mais comum em proteções pessoais. Atualmente, a evolução das ameaças balísticas no campo de batalha requer o desenvolvimento contínuo de armaduras cerâmicas de melhor desempenho. Um exemplo disso são as cerâmicas ligadas por reação (reactionbonded) que foram desenvolvidas e empregadas para aplicações de armadura balística. As cerâmicas comerciais mais utilizadas e seus respectivos processamentos estão na Tabela II (adaptada [7]):

Esses processamentos definem as propriedades das cerâmicas balísticas, seu custo e sua viabilidade econômica no desenvolvimento de um sistema de proteção efetivo.

\section{Cerâmicas de alumina}

A alumina é um material estrutural de engenharia largamente utilizado com a melhor relação custo/benefício das cerâmicas avançadas. As matérias-primas utilizadas na produção desta cerâmica técnica de alto desempenho são de fácil disponibilidade e de custo relativamente baixo. Assim, a alumina em sua fase $\alpha$ (coríndon) é considerada o material de serviço por excelência da indústria da cerâmica industrial. Sua dureza, resistência à abrasão e inércia química fazem-na um material ideal para utilizações em ambientes agressivos, desde indústrias de mineração, de vidros e armaduras balísticas, até aplicações biomédicas.

Entretanto, sua alta densidade e sua baixa resistência à flexão e dureza em comparação a outras cerâmicas balísticas fazem com que o uso de peças de alumina em projetos onde o fator peso é crucial deva ser meticulosamente avaliado com o intuito de evitar falhas catastróficas. Uma análise das principais propriedades nominais da alumina é mostrada na Tabela III (adaptada [10]):

Tabela III - Propriedades gerais das cerâmicas de alumina. [Table III - General properties of alumina ceramics.]

\begin{tabular}{lc}
\hline Propriedades & $\begin{array}{c}\text { Intervalo de } \\
\text { valores }\end{array}$ \\
\hline Densidade relativa $\left(\mathrm{g} / \mathrm{cm}^{3}\right)$ & $3,4-3,7$ \\
Coeficiente de expansão térmica $\left(\times 10^{-6}{ }^{\circ} \mathrm{C}^{-1}\right)$ & $7,5-8,5$ \\
Resistência à compressão $(\mathrm{MPa})$ & $1000-2800$ \\
Resistência à tração $(\mathrm{MPa})$ & $140-170$ \\
Resistência à flexão $(\mathrm{MPa})$ & $280-420$ \\
Tenacidade à fratura $\left(\mathrm{MPa} \cdot \mathrm{m}^{1 / 2}\right)$ & $3-4$ \\
Módulo elástico $(\mathrm{GPa})$ & $350-400$ \\
Módulo de cisalhamento $(\mathrm{GPa})$ & $140-160$ \\
Microdureza $\left(\mathrm{kg} \cdot \mathrm{mm}^{-2}\right)$ & $1400-1800$ \\
\hline
\end{tabular}

A grande faixa de valores de propriedades atesta o fato de que as cerâmicas de alumina são dependentes dos parâmetros de processamento, como temperatura e atmosfera de sinterização, impurezas, tamanho de grão e outros fatores extrínsecos e intrínsecos. Por exemplo, alumina de extrema pureza e com grãos muito refinados é utilizada em cabeças femorais para endopróteses de quadril, apresentando valores de resistência mecânica, tenacidade e dureza notavelmente mais elevados. A alumina utilizada em blindagens mistas apresenta como principais vantagens sua relação custo/ benefício e sua disponibilidade, apresentando bom desempenho balístico. Suas desvantagens residem no fato de ser a cerâmica balística de maior densidade (limitando o uso em proteção pessoal) e suas propriedades de tenacidade a fratura e resistência a flexão serem baixas (limitando os 
Tabela IV - Propriedades das cerâmicas comerciais de alumina para proteção balística.

[Table IV - Properties of commercial alumina ceramics for ballistic protection.]

\begin{tabular}{cccccccccc}
\hline Material & Denominação & $\begin{array}{c}\text { Densidade } \\
\left(\mathrm{g} / \mathrm{cm}^{3}\right)\end{array}$ & $\begin{array}{c}\text { Tamanho } \\
\text { de grão } \\
(\mu \mathrm{m})\end{array}$ & $\begin{array}{c}\text { Módulo Resistência aResistência a } \\
(\mathrm{GPa})\end{array}$ & $\begin{array}{c}\text { Flexão } \\
(\mathrm{MPa})\end{array}$ & $\begin{array}{c}\text { Fratura } \\
\left(\mathrm{MPa} \bullet \mathrm{m}^{1 / 2}\right)\end{array}$ & $\begin{array}{c}\text { Dureza } \\
\left(\mathrm{kg} / \mathrm{mm}^{2}\right)\end{array}$ & $\begin{array}{c}\mathrm{HK} 2 \mathrm{~kg} \\
\left(\mathrm{~kg} / \mathrm{mm}^{2}\right)\end{array}$ & $\begin{array}{c}\text { Densidade de } \\
\text { painel* } \\
\left(\mathrm{g} / \mathrm{cm}^{2}\right)\end{array}$ \\
\hline $\mathrm{Al}_{2} \mathrm{O}_{3}$ & CAP-3 & 3,90 & -- & 370 & 379 & $4-5$ & $\begin{array}{c}1440(\mathrm{HK} \\
1 \mathrm{~kg})\end{array}$ & 1292 & 9,9 \\
\hline
\end{tabular}

$* 12 " \times 12 " \times 1 "(30,5 \mathrm{~cm} \times 30,5 \mathrm{~cm} \times 2,54 \mathrm{~cm})$

níveis de proteção) em comparação as outras cerâmicas balísticas. A Tabela IV (adaptada [7]) mostra as propriedades das cerâmicas balísticas comerciais de alumina.

\section{Cerâmicas de carbeto de boro}

$\mathrm{O}$ carbeto de boro $\left(\mathrm{B}_{4} \mathrm{C}\right)$ é caracterizado por uma combinação incomum de propriedades que resultam em sua utilização em um grande número de aplicações. $\mathrm{O} \mathrm{B}_{4} \mathrm{C}$ apresenta propriedades refratárias devido a seu elevado ponto de fusão $\left(2450{ }^{\circ} \mathrm{C}\right)$ e sua estabilidade térmica. Tem altíssima dureza (HV 25-35 GPa) sendo superado apenas pelo diamante e pelo nitreto cúbico de boro, além de elevado módulo de elasticidade (450 GPa) e uma das menores densidades entre as cerâmicas avançadas $\left(2,52 \mathrm{~g} / \mathrm{cm}^{3}\right)$ [10]. Essas características definem muitas de suas aplicações, incluindo bicos injetores para outros materiais abrasivos como alumina e carbeto de silício, ferramentas de usinagem, elementos de moagem e polimento, entre muitas outras. Suas propriedades de baixa densidade e elevada dureza explicam sua utilização em armaduras balísticas, tanto pessoais quanto veiculares. Normalmente, $\mathrm{B}_{4} \mathrm{C}$ é produzido por redução carbotérmica de trióxido de boro com coque em um forno de arco elétrico. Corpos densos podem ser obtidos após purificação e cominuição, seguida de prensagem a quente (hot pressing), prensagem isostática a quente (hot isostatic pressing), ou sinterização a vácuo em temperaturas superiores a $2000{ }^{\circ} \mathrm{C}$ com uso de aditivos. A Tabela V (adaptada [7]) apresenta as propriedades mecânicas das cerâmicas de carbeto de boro:

\section{Cerâmicas de carbeto de silício}

O carbeto de silício foi descoberto em 1890 quando se estudava síntese de diamantes. Ocorrendo raramente na natureza na forma do mineral moissanita, o carbeto de silício é produzido em grandes volumes desde o final do século XIX, principalmente para uso como abrasivo. Há muito tempo encontra aplicações em operações de usinagem, moagem, polimento, jateamento com areia e corte a jato d'água. Atualmente, outras aplicações se beneficiam de sua alta dureza, condutividade térmica, estabilidade química e propriedades semicondutoras. Dentre essas, inclui-se a proteção balística para veículos e pessoas na forma de blindagens mistas. Sua principal vantagem está em suas propriedades superiores a da alumina (menor densidade e maior dureza) e custo inferior ao do carbeto de boro. Assim, é uma escolha relativamente mais acessível ao combinar boas propriedades e a um custo mais baixo. Devido a essas características, o carbeto de silício é um dos materiais mais utilizados no desenvolvimento de vestes balísticas apresentando um grande número de produtos, conforme a Tabela VI (adaptada [7]).

\section{Cerâmicas de alumina-zircônia tetragonal}

A melhoria das qualidades da alumina utilizando zircônia tetragonal policristalina (tetragonal zirconia polycrystals, TZP) tem sido alvo de pesquisa em várias partes do mundo, devido principalmente a seu mecanismo de tenacificação por transformação da zircônia $[11,12]$. A transformação de fase tetragonal para monoclínica da zircônia apresenta um caráter martensítico, ou seja, um processo adifusional de extrema velocidade que é caracterizado por uma histerese de transformações durante ciclos de aquecimento ou resfriamento. A taxa da transformação martensítica e seus mecanismos são muito similares àqueles encontrados

Tabela V - Propriedades das cerâmicas comerciais de carbeto de boro para proteção balística.

[Table V-Properties of commercial boron carbide ceramics for ballistic protection.]

\begin{tabular}{cccccccccc}
\hline Material & Denominação & $\begin{array}{c}\text { Densidade } \\
\left(\mathrm{g} / \mathrm{cm}^{3}\right)\end{array}$ & $\begin{array}{c}\text { Tamanho de } \\
\text { grão }(\mu \mathrm{m})\end{array}$ & $\begin{array}{c}\text { Módulo Young } \\
(\mathrm{GPa})\end{array}$ & $\begin{array}{c}\text { Resistência } \\
\text { a Flexão }(\mathrm{MPa})\end{array}$ & $\begin{array}{c}\text { Fesistência a } \\
\mathrm{K}_{\mathrm{Ic}} \\
\left(\mathrm{MPa} \bullet \mathrm{m}^{1 / 2}\right)\end{array}$ & $\begin{array}{c}\text { Dureza } \\
\left(\mathrm{kg} / \mathrm{mm}^{2}\right)\end{array}$ & $\begin{array}{c}\mathrm{HK} 2 \mathrm{~kg} \\
\left(\mathrm{~kg} / \mathrm{mm}^{2}\right)\end{array}$ & $\begin{array}{c}\text { Densidade } \\
\text { de painel* } \\
\left(\mathrm{g} / \mathrm{cm}^{2}\right)\end{array}$ \\
\hline $\mathrm{B}_{4} \mathrm{C}$ & $\begin{array}{c}\text { Ceralloy-566 } \\
4 \mathrm{E}\end{array}$ & 2,50 & $10-15$ & 460 & 410 & 2,5 & $\begin{array}{c}3200 \\
(\mathrm{HV} \mathrm{0,3} \mathrm{kg}) \\
2800\end{array}$ & 2066 & 6,3 \\
& Norbide & 2,51 & $10-15$ & 440 & 425 & 3,1 & $\begin{array}{c}2006 \\
(\mathrm{HK} 0,1 \mathrm{~kg})\end{array}$ & 1997 & 6,3 \\
\hline
\end{tabular}


Tabela VI - Propriedades das cerâmicas comerciais de carbeto de silício para proteção balística. [Table VI - Properties of commercial silicon carbide ceramics for ballistic protection.]

\begin{tabular}{|c|c|c|c|c|c|c|c|c|c|}
\hline Material & Denominação & $\begin{array}{c}\text { Densidade } \\
\left(\mathrm{g} / \mathrm{cm}^{3}\right)\end{array}$ & $\begin{array}{c}\text { Tamanho } \\
\text { de grão } \\
(\mu \mathrm{m})\end{array}$ & $\begin{array}{c}\text { Módulo } \\
\text { de Young } \\
(\mathrm{GPa})\end{array}$ & $\begin{array}{c}\text { Resistência } \\
\text { a Flexão } \\
(\mathrm{MPa})\end{array}$ & $\begin{array}{c}\text { Resistência } \\
\text { a Fratura } \\
\left(\mathrm{MPa} \cdot \mathrm{m}^{1 / 2}\right)\end{array}$ & $\begin{array}{c}\text { Dureza } \\
\left(\mathrm{kg} / \mathrm{mm}^{2}\right)\end{array}$ & $\begin{array}{c}\mathrm{HK} 2 \mathrm{~kg} \\
\left(\mathrm{~kg} / \mathrm{mm}^{2}\right)\end{array}$ & $\begin{array}{c}\text { Densidade } \\
\text { de painel* } \\
\left(\mathrm{g} / \mathrm{cm}^{2}\right)\end{array}$ \\
\hline \multirow[t]{8}{*}{$\mathrm{SiC}$} & SiC-N & 3,22 & $2-5$ & 453 & 486 & 4,0 & -- & 1905 & 8,2 \\
\hline & $\begin{array}{c}\text { Ceralloy 146- } \\
3 \mathrm{E}\end{array}$ & 3,20 & -- & 450 & 634 & 4,3 & $2300(\mathrm{HV} 0,3 \mathrm{~kg})$ & -- & 8,1 \\
\hline & Hexoloy & 3,13 & $3-50$ & 410 & 380 & 4,6 & $2800(\mathrm{HK} 0,1 \mathrm{~kg})$ & 1924 & 7,9 \\
\hline & Purebide 5000 & 3,10 & $3-50$ & 420 & 455 & -- & -- & 1922 & 7,9 \\
\hline & SC-DS & 3,15 & $3-50$ & 410 & 480 & $3-4$ & $2800(\mathrm{HK} 0,1 \mathrm{~kg})$ & - & 8,0 \\
\hline & MCT SSS & 3,12 & $3-50$ & 424 & 351 & 4,0 & -- & 1969 & 7,9 \\
\hline & MCT LPS & 3,24 & $1-3$ & 425 & 372 & 5,7 & -- & 1873 & 8,2 \\
\hline & Ekasic-T & 3,25 & $1-3$ & 453 & 612 & 6,4 & -- & 1928 & 8,2 \\
\hline \multirow[t]{3}{*}{$\operatorname{SiC}(\mathrm{RB})$} & SSC-702 & 3,02 & 45 & 359 & 260 & 4,0 & 1757 (HK 0,5 kg) & -- & 7,7 \\
\hline & SSC-802 & 3,03 & 45 & 380 & 260 & 4,0 & -- & 1332 & 7,7 \\
\hline & SSC-902 & 3,12 & 45 & 407 & 260 & 4,0 & -- & 1536 & 7,9 \\
\hline $\begin{array}{c}\mathrm{SiC} / \mathrm{B}_{4} \mathrm{C} \\
(\mathrm{RB})\end{array}$ & RBBC-751 & 2,56 & 45 & 390 & 271 & 5,0 & -- & 1626 & 6,5 \\
\hline
\end{tabular}

durante a transformação do ferro $\alpha$ (CCC) para ferro $\gamma$ (CFC). Entretanto, há uma diferença muito importante entre a transformação martensítica de materiais inerentemente dúcteis como o ferro e materiais frágeis como a zircônia, já que a transformação normalmente resulta em grandes modificações de volume e os valores de limite elástico e limite de resistência da zircônia pura não suportam tamanha mudança, gerando trincas catastróficas. A adição dos óxidos de ítrio, magnésio, cálcio e cério retardam a transformação, pois a carga destes cátions normalmente é inferior à do íon $\mathrm{Zr}^{4+}$, o que ocasiona a formação de vacâncias na matriz (motivo de sua condutividade iônica) [13].

A teoria clássica de Griffith-Orowan descreve a relação entre resistência mecânica e tenacidade de materiais frágeis como as cerâmicas. Na equação básica da teoria, a tensão de fratura $\sigma_{\mathrm{f}}$ é relacionada ao módulo de elasticidade $\mathrm{E}$, à energia de fratura $\gamma$ e ao comprimento crítico de trinca c pela equação A:

$$
\sigma_{\mathrm{f}}=\sqrt{(2 \mathrm{E} \gamma / \pi \mathrm{c})}
$$

A tenacidade à fratura $\mathrm{K}_{\mathrm{Ic}}$, por sua vez, é dada por:

$$
\mathrm{k}_{\mathrm{Ic}}=\sqrt{2 \mathrm{E} \cdot \gamma}
$$

A tensão a fratura então se torna:

$$
\sigma_{\mathrm{f}}=\frac{1}{\sqrt{\pi}} \cdot \mathrm{k}_{\mathrm{Ic}} \cdot \mathrm{c}^{-1 / 2}
$$

Assim uma trinca de comprimento crítico é capaz de se mover através do campo de tensões, mas será defletida pelas partículas que se transformarem espontaneamente para a forma monoclínica nas proximidades de uma trinca progressiva (Fig. 3).
A energia da trinca será absorvida e a mudança de volume irá prevenir a continuidade do avanço da trinca. Isso se expressa em um aumento da tenacidade à fratura da zircônia parcialmente estável pelo então chamado mecanismo de tenacificação por transformação. Assim, as propriedades mecânicas de materiais desenvolvidos com zircônia não serão limitadas por falhas induzidas no processo, pois existe tolerância a dano devido ao comportamento de deformação plástica [10]. Com a propagação da trinca, inicialmente ocorrem formações de trincas subcríticas em torno de um

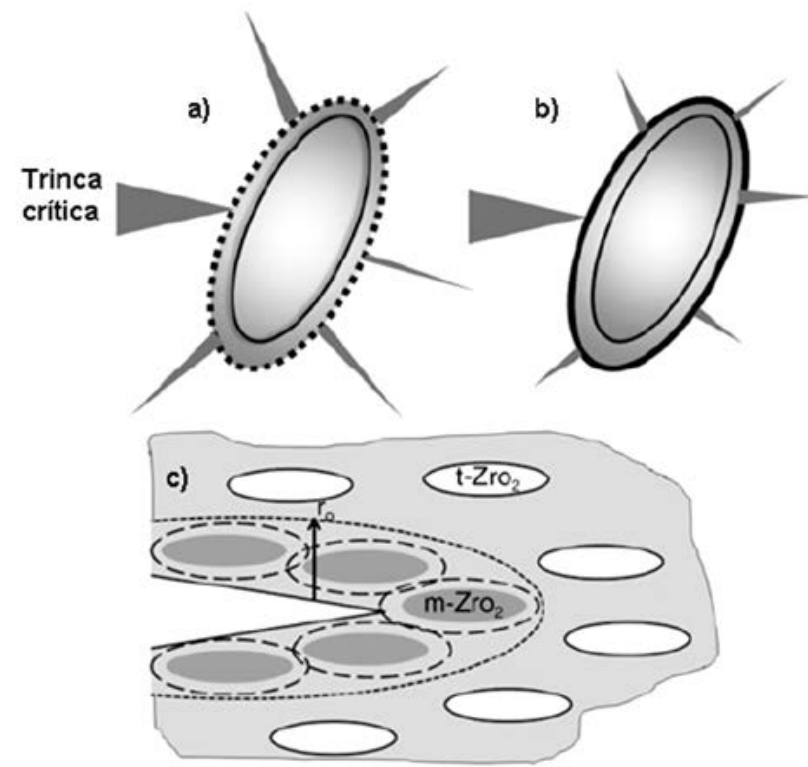

Figura 3: Mecanismo de tenacificação por transformação da zircônia estabilizada.

[Figure 3: Mechanism of toughening by transformation of stabilized zirconia.] 
grão de zircônia metaestável. Inicialmente, Figs. 3a-b, esse grão sofre transformação de estado cristalino de tetragonal para monoclínico $(\mathrm{t} \rightarrow \mathrm{m})$ gerando expansão de volume e tensões cisalhantes que detém o movimento da trinca. Em uma visão mais abrangente (Fig. 3c) observa-se uma trinca penetrando uma matriz de zircônia monoclínica com zircônia tetragonal incorporada $\left(\mathrm{t}-\mathrm{ZrO}_{2}\right)$. A trinca gera em torno de sua ponta um campo de tensões de raio $r_{0}$ que ao liberar a tensão da matriz na fase tetragonal desencadeia uma transformação martensítica $(\mathrm{t} \rightarrow \mathrm{m})$. A expansão de volume (3 a 5\%) e as tensões de cisalhamento existentes (1 a $7 \%$ ) causam tensões compressivas em torno da extremidade da trinca, detendo seu movimento.

Compósitos cerâmicos de alumina/zircônia tetragonal (ZTA) combinam propriedades das duas cerâmicas, como resistência alta ao desgaste abrasivo e erosivo, elevada estabilidade química, e alta tenacidade à fratura. Por isso, são atraentes para uso na indústria aeroespacial, apresentando grande potencial para a produção de componentes mecânicos como válvulas, filtros, orifícios, cilindros, rotores e proteções de foguetes e mísseis [2].

O compósito policristalino de alumina-zircônia resulta num aumento da resistência à flexão, da tenacidade à fratura e da resistência à fadiga em relação à alumina pura [11]. Nesse caso, as partículas de alumina estão sujeitas a campos de tensões compressivas devido ao seu baixo coeficiente de expansão térmica. Além disso, há aumento de volume das partículas de zircônia na sua transformação de fase tetragonal para monoclínica, como discutido anteriormente. A Fig. 4 (adaptada [12]) apresenta um modelo da microestrutura de um compósito ZTA.

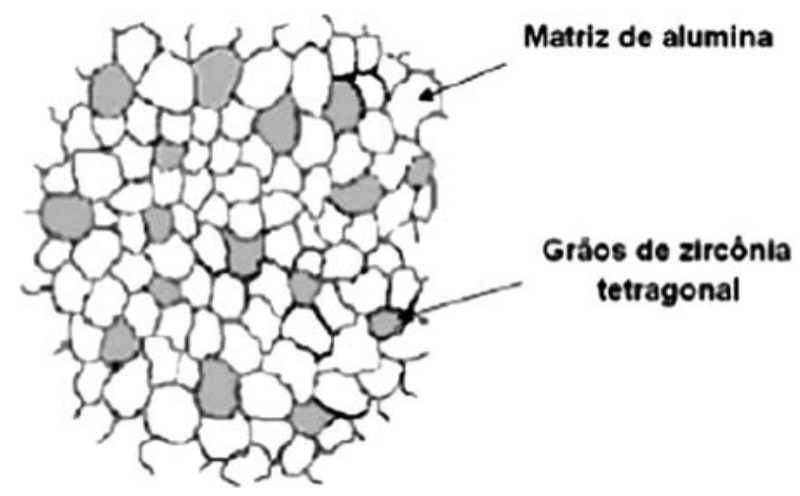

Figura 4: Modelo da microestrutura de um compósito cerâmico de matriz de alumina com grãos de zircônia.

[Figure 4: Model of the microstructure of ceramic matrix composite with alumina matrix and zirconia grains.]

A zircônia possui baixa solubilidade em alumina, conforme diagrama de fases do sistema $\mathrm{Al}_{2} \mathrm{O}_{3} / \mathrm{ZrO}_{2}$ (Fig. 5 , adaptada [14]). Aqui pode ser observada uma pequena região de solubilidade da zircônia na alumina $(\sim 1 \%)$ em temperaturas acima de $1600{ }^{\circ} \mathrm{C}$ formando uma solução sólida. As demais composições químicas resultam apenas em uma mistura de $\mathrm{Al}_{2} \mathrm{O}_{3}$ e $\mathrm{ZrO}_{2}$. Um ponto eutético está localizado em $42,5 \%$ em massa de $\mathrm{ZrO}_{2}$ a $1910{ }^{\circ} \mathrm{C}$.

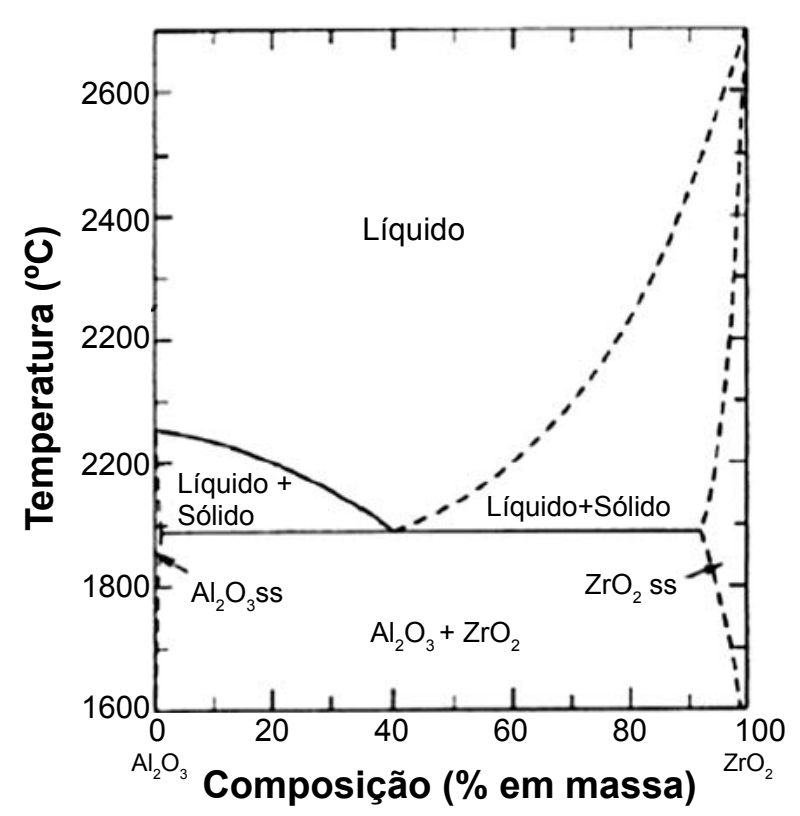

Figura 5: Diagrama de equilíbrio de fases no sistema $\mathrm{Al}_{2} \mathrm{O}_{3}-\mathrm{ZrO}_{2}$. [Figure 5: Phase equilibrium diagram of $\mathrm{Al}_{2} \mathrm{O}_{3}-\mathrm{ZrO}_{2}$.]

Dependendo da quantidade de ítria adicionada ao sistema, a zircônia estabiliza nas formas tetragonal ou cúbica, apresentando dois pontos eutéticos no diagrama de fases ternário $\left(\mathrm{Al}_{2} \mathrm{O}_{3} / \mathrm{ZrO}_{2}\right.$ e $\left.\mathrm{Y}_{2} \mathrm{O}_{3}\right)$. Quando se adiciona 3 mol\% de ítria, a zircônia apresenta estrutura tetragonal e há um ponto eutético em $33 \mathrm{~mol} \%$. Na adição de $9 \mathrm{~mol} \%$ de ítria, a zircônia apresenta estrutura cúbica, e um ponto eutético em $35 \mathrm{~mol} \%$ [14].

Estudos mostraram que as cerâmicas solidificadas no ponto eutético possuíam propriedades superiores que a dos seus vizinhos devido à forte compressão de sua microestrutura. Na temperatura ambiente, apresentaram alta resistência ao desgaste. Neste ponto, a tenacidade à fratura é máxima, embora o aumento de tenacidade tenha implicado na diminuição de outras propriedades mecânicas [15].

\section{Compósitos de Matriz Cerâmica}

Compósitos de matriz cerâmica (CMCs) consistem de uma matriz cerâmica com elementos de reforço (geralmente fibras), também de natureza cerâmica. Os materiais da matriz e da fibra podem ser carbono $(\mathrm{C})$, carbeto de silício $(\mathrm{SiC})$, alumina $\left(\mathrm{Al}_{2} \mathrm{O}_{3}\right)$ e mulita $\left(\mathrm{Al}_{2} \mathrm{O}_{3}-\mathrm{SiO}_{2}\right)$, dentre outros.

Materiais porosos apresentando estrutura celular ou fibrosa, por exemplo, são tipicamente leves, isolantes térmicos e facilmente deformáveis elasticamente quando comparados com materiais densos. Materiais cerâmicos são em geral frágeis, e, de acordo com a mecânica da fratura, sua resistência é definida pelo tamanho dos defeitos (microtrincas e poros) e pela tenacidade à fratura. Trabalhar com defeitos é limitado pela natureza da microestrutura das cerâmicas, onde um contorno de grão pode ser responsável por uma fratura frágil. Defeitos superficiais gerados em serviço também reduzem a resistência das cerâmicas. Um esforço considerável tem sido feito para se desenvolver 

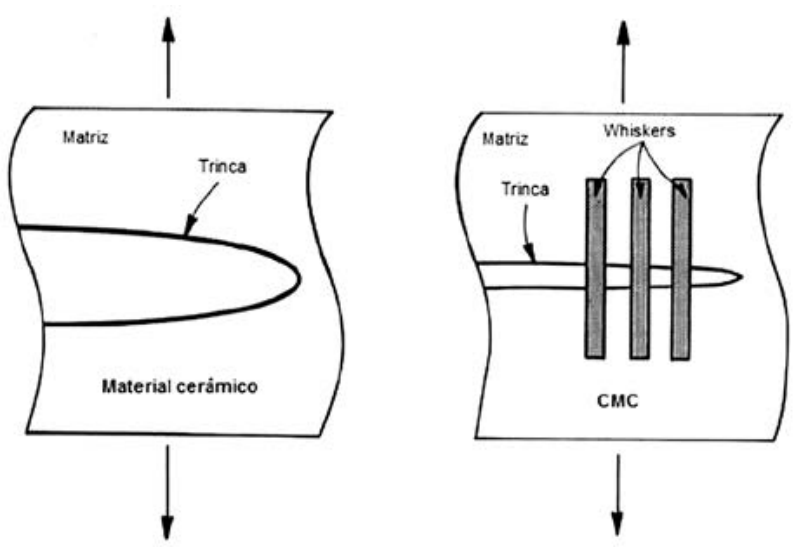

Figura 6: Exemplo de um material cerâmico e de um CMC sujeitos a tensões e trincas.

[Figure 6: Example of a ceramic material and a CMC subjected to stresses and cracks.]

cerâmicas mais resistentes com alta tenacidade à fratura [2].

A tenacidade das cerâmicas é melhorada por meio da introdução de fases secundárias nas matrizes de determinados materiais, as quais agem como barreiras para a propagação de trincas. A introdução de whiskers (monocristais alongados) ou fibras em uma matriz cerâmica, por exemplo, pode retardar a propagação de trincas (aumentando a tenacidade), pois se necessita de maior tensão para se propagar trincas por whiskers ou fibras, conforme a Fig. 6 (adaptada [16]).

Os compósitos de matriz cerâmica podem ser classificados em dois grupos. O primeiro tem como elementos de reforço partículas ou whiskers, os quais exibem comportamento frágil mesmo com aumentos consideráveis de tenacidade à fratura e resistência mecânica. $\mathrm{O}$ segundo grupo consiste em compósitos com fibras contínuas que exibem um comportamento de trinca quase dúctil acompanhado de fiber pullout (tipo de falha em compósitos com fibra onde as fibras "escorregam" pelo material matriz quando submetidas a trincas). A Fig. 7 [17] exemplifica tal comportamento.

Normalmente, a nomenclatura de CMCs inclui uma combinação do tipo de fibra/tipo de matriz, por exemplo, $\mathrm{C} / \mathrm{C}$ significa carbono reforçado com fibras de carbono ou $\mathrm{C} /$ $\mathrm{SiC}$ para carbeto de silício reforçado com fibras de carbono. $\mathrm{O}$ processo de fabricação também pode ser incluído e um

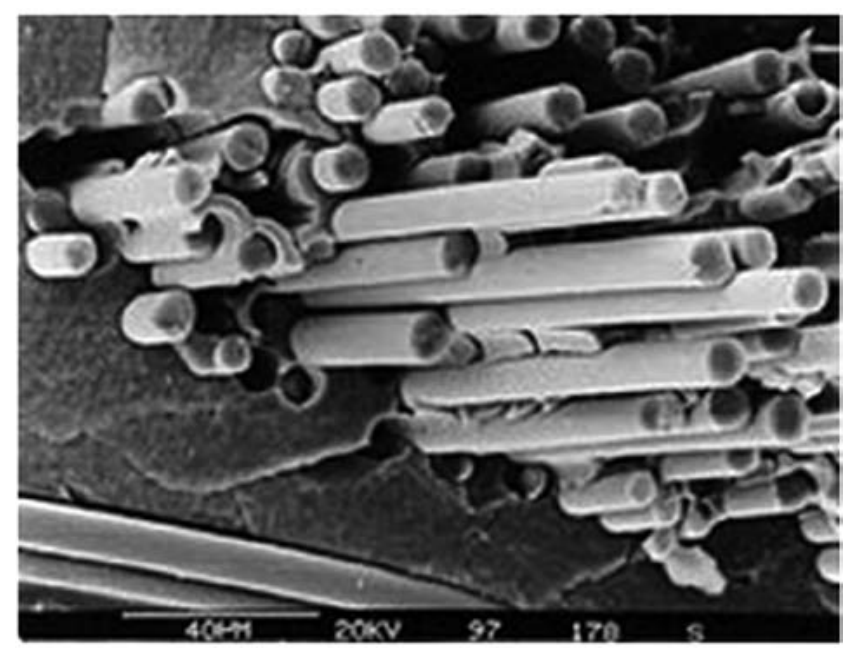

Figura 7: Exemplo de fiber pullout em compósitos de matriz cerâmica.

[Figure 7: Example of fiber pullout in ceramic matrix composites.]

compósito $\mathrm{C} / \mathrm{SiC}$ produzido por infiltração de polímero líquido (liquid polymer infiltration, LPI) é abreviado para LPI-C/SiC. A Tabela VII (adaptada [17]) apresenta as propriedades dos mais comuns compósitos de matriz cerâmica.

Os CMCs mais importantes, tanto do ponto de vista de investigação quanto de disponibilidade comercial são $\mathrm{C} / \mathrm{C}$ [18], C/SiC [19], $\mathrm{SiC} / \mathrm{SiC}$ [20] e $\mathrm{Al}_{2} \mathrm{O}_{3} / \mathrm{Al}_{2} \mathrm{O}_{3}$ [21]. Os CMCs se diferem de suas cerâmicas convencionais nas seguintes propriedades: alongamento de até $1 \%$ antes da ruptura; tenacidade à fratura consideravelmente mais elevada; elevada resistência a choque térmico; capacidade de carregamento dinâmico aprimorada; propriedades anisotrópicas seguindo a orientação das fibras.

O modelamento e a simulação do comportamento de compósitos de matriz cerâmica têm avançado. Para feixes de fibras, a força do feixe é sempre menor do que a soma das forças de cada fibra. Este comportamento pode ser explicado pelo modelo carga de compartilhamento de carga. Quando a fibra mais fraca falha, as fibras restantes compartilham a carga, aumentando assim a tensão efetiva nessas fibras [22].

Novos sistemas de proteção balística com base em materiais alternativos têm sido desenvolvidos recentemente.

Tabela VII - Propriedades dos compósitos mais comuns de matriz cerâmica.

[Table VII - Properties of most common ceramic matrix composites.]

\begin{tabular}{lccccc}
\hline \multicolumn{1}{c}{ Material* } & $\mathrm{Al}_{2} \mathrm{O}_{3} / \mathrm{Al}_{2} \mathrm{O}_{3}$ & $\mathrm{CVI}-\mathrm{C} / \mathrm{SiC}$ & LPI-C/SiC & LSI-C/SiC & SiSiC \\
\hline Porosidade (\%) & 35 & 12 & 12 & 3 & $<1$ \\
Densidade $\left(\mathrm{g} / \mathrm{cm}^{3}\right)$ & 2,1 & 2,1 & 1,9 & 1,9 & 3,1 \\
Resistência à Tração (MPa) & 65 & 310 & 250 & 190 & 200 \\
Alongamento (\%) & 0,12 & 0,75 & 0,5 & 0,35 & 0,05 \\
Módulo de Young (GPa) & 50 & 95 & 65 & 60 & 395 \\
Resistência à Flexão (MPa) & 80 & 475 & 500 & 300 & 400 \\
\hline
\end{tabular}

*CVI (chemical vapor infiltration); LPI (liquid polymer infiltration); LSI (liquid silicon infiltration). 
Um dos objetivos da indústria é desenvolver sistemas defensivos mais leves e mais fortes, que permitam maior mobilidade e segurança para veículos e pessoas. Em um modelamento [23] foi estudado o comportamento de um sistema de proteção cerâmica-metal contra um impacto de projétil, buscando uma construção otimizada. $\mathrm{O}$ avanço no conhecimento dos processos de fratura por impacto de alta velocidade tem colaborado para o desenvolvimento de novos materiais para a absorção de choque.

\section{CONSIDERAÇÕES FINAIS}

Materiais cerâmicos podem ser usados de forma simples ou combinada com materiais metálicos e/ou poliméricos em sistemas de proteção balística. As cerâmicas mais utilizadas para esta finalidade são alumina e carbetos de boro e de silício. Novas técnicas de processamento de materiais e compósitos cerâmicos, além do modelamento do mecanismo de fratura, têm sido amplamente investigados. Desenvolvimentos em particular na área de compósitos de matriz cerâmica reforçados com fibras ou com base na transformação por tenacificação abrem novas perspectivas de uso e de melhor desempenho de sistemas de proteção balística a base de materiais cerâmicos.

\section{REFERÊNCIAS}

[1] S. Yadav, G. Ravichandran, Int. J. Impact Eng. 28 (2003) 557.

[2] J. LaSalvia, "Advances in Ceramic Armor", Wiley, New York, EUA (2013).

[3] Matweb, Alumina. Disponível em http://www.matweb. com/search, acesso em 12/2013.

[4] M. L. Wilkins, Int. J. Eng. Sci. 16 (1978) 793.

[5] S. M. Lee, "International Encyclopedia of Composites", VCH, New York, EUA (1991).

[6] C. Xavier, C. R. C. Costa, Cerâmica 30, 175 (1984) 161.
[7] P. G. Karandikar, G. Evans, S. Wong, M. K. Aghajanian, M. Sennett, Ceram. Eng. Sci. Proc. 29 (2009) 163.

[8] R. E. Tressler, Ceram. Trans. 134 (2002) 451.

[9] R. Stevens, A. G. Evans, Brit. Ceram. Trans. J. 83 (1984) 18.

[10] R. B. Heimann, "Classic and Advanced Ceramics: From Fundamentals to Applications", Wiley-VCH, Weinhein, 2010.

[11] M. Ruhle, N. Claussen, A. H. Hever, J. Am. Ceram. Soc. 69 (1986) 195.

[12] A. C. O. Hirschmann, "Compósitos cerâmicos porosos de alumina-zircônia para aplicação em sistemas de controle térmico para satélites: obtenção e caracterização", Tese Dr., Ciência e Tecnologia de Materiais e Sensores, INPE, S. José dos Campos, SP (2008) 140p.

[13] G. R. Fischer, L. H. Manfredo, R. N. McNally, R. C. Doman, J. Mater. Sci. 16 (1981) 3447.

[14] L. Mazerolles, D. Michel, R. Portier, J. Am. Ceram. Soc. 69 (1986) 252.

[15] K. Miyoshi, S. C. Farmer, A. Saiyr, Tribology Int. 38 (2005) 974.

[16] M. Kuntz, Ceram. Forum Int. 49 (1992) 18.

[17] W. Krenkel, "Ceramic Matrix Composites", Wiley$\mathrm{VCH}$, Weinhein (2009).

[18] E. Fitzer, Carbon 25 (1987) 163.

[19] W. Krenkel, B. Heidenreich, R. Renz, Adv. Eng. Mater. 4 (2002) 427.

[20] A. Kohyama, Y. Katoh, Ceram. Trans. 144 (2003) 3.

[21] S. M. Goushegir, P. O. Guglielmi, J. G. P. Silva, M. P. Hablitzel, D. Hotza, H. A. Al-Qureshi, J. Am. Ceram. Soc. 95 (2012) 159.

[22] J. G. P. Silva, D. Hotza, R. Janssen, H. A. Al-Qureshi, Mater. Sci. Forum 727-728 (2012) 574.

[23] L. Neckel, D. Hotza, D. Stainer, R. Janssen, A. G. R. Lezana, A. Dias, H. A. Al-Qureshi, Key Eng. Mater. 488489 (2012) 25-28.

(Rec. 17/06/2013, Rev. 08/03/2014, Ac. 09/03/2014) 